\title{
Morphological study of the TK cholangiocarcinoma cell line with three-dimensional cell culture
}

\author{
KOHEI AKIYOSHI ${ }^{1}$, MINORI KAMADA ${ }^{2}$, NOBUTAKE AKIYAMA ${ }^{3}$, MASAFUMI SUZUKI $^{4}$, \\ MICHIKO WATANABE ${ }^{5}$, KOUKI FUJIOKA ${ }^{1}$, KEIICHI IKEDA ${ }^{1}$, SHUICHI MIZUNO ${ }^{6}$ and YOSHINOBU MANOME $^{1}$ \\ ${ }^{1}$ Department of Molecular Cell Biology, ${ }^{2}$ Institute of DNA Medicine, ${ }^{3}$ Department of Molecular Immunology, \\ Research Center for Medical Sciences, and ${ }^{4}$ Department of Pathology, Jikei University School of Medicine, \\ Minato, Tokyo 105-8461; ${ }^{5}$ Institute of DNA Sciences, Yokohama, Kanagawa 231-0862, Japan; ${ }^{6}$ Department of \\ Orthopedic Surgery, Brigham and Women's Hospital, Harvard Medical School, Boston, MA 02115, USA
}

Received May 27, 2013; Accepted January 23, 2014

DOI: $10.3892 / \mathrm{mmr} .2014 .1939$

\begin{abstract}
Cholangiocarcinoma is an intractable carcinoma originating from the bile duct epithelium. To gain an understanding of the cell biology of cholangiocarcinoma, in vitro cell culture is valuable. However, well-characterized cell lines are limited. In the present study, the morphology of the TK cholangiocarcinoma cell line was analyzed by three-dimensional culture. Dispersed TK cells were injected into a gelatin mesh scaffold and cultivated for 3-20 days. The morphology of the TK cells was investigated by phase-contrast microscopy, optical microscopy, scanning electron microscopy (SEM) and transmission electron microscopy (TEM). TK cells were observed to proliferate three-dimensionally in the scaffold. The cells exhibited a globoid structure and attached to the scaffold. The SEM observation demonstrated typical microvilli and plicae on the surface of the structure. Light microscopy and TEM confirmed intercellular and cell-to-scaffold attachment in the three-dimensional mesh. The culture also exhibited the formation of a duct-like structure covered by structured microvilli. In conclusion, three-dimensional culture of TK cells demonstrated the morphological characteristics of cholangiocarcinoma in vitro. Production of high levels of carbohydrate antigen (CA)19-9, CA50 and carcinoembryonic antigen was previously confirmed in the TK cell line. As a characteristic morphology was demonstrated in the present study, the TK cholangiocarcinoma cell line may be useful as an experimental model for further study of cholangiocarcinoma.
\end{abstract}

Correspondence to: Professor Yoshinobu Manome, Department of Molecular Cell Biology, Jikei University School of Medicine, 3-25-8 Nishishimbashi, Minato-ku, Tokyo 105-8461, Japan

E-mail: manome@jikei.ac.jp

Key words: cholangiocarcinoma, cell line, three-dimensional culture, morphology

\section{Introduction}

Cholangiocarcinoma is an intractable malignant tumor originating from the bile duct, and the incidence of this carcinoma is increasing worldwide. Regardless of recent advances in medical treatment, surgical resection is the only potentially curative treatment for the disease. However, less than one-third of patients recommended for surgical resection as the tumor rapidly progresses to the perivascular or organ systems and is often only diagnosed at an advanced stage. Although chemotherapy, including a combination of molecular targeting-therapies, is the therapy of choice for the majority of patients, the prognosis remains poor at the present time $(1,2)$. While most patients do not have a specific background underlying the occurrence of cholangiocarcinoma, several risk factors have been demonstrated in the disease pathogenesis (3). These factors include the presence of gallstones, chronic ulcerative colitis, infection by liver flukes, congenital biliary cysts, primary sclerosing cholangitis (PSC) and nitrosamine exposure $(4,5)$.

Recently, cholangiocarcinoma has been frequently highlighted as a high incidence was observed amongst industrial workers in Japanese printing companies (6). Five cholangiocarcinoma cases were identified among 33 workers who were employed for $>1$ year in a printing factory in Osaka. Further investigation increased the total case load to 18 . In this instance, the onset of the disease was at a relatively young age, arising between the ages of 25-40 years. Similar cases were also identified in the Tokyo, Fukuoka and Miyagi provinces in Japan. Etiology of the disease has been attributed to long-term exposure to chemical substances, such as dichloromethane and 1,2-dichloropropane (7). However, further investigation is required to clarify the involvement of these chemicals in the pathogenesis of cholangiocarcinoma, including tumor initiation, promotion and/or progression.

In order to understand the mechanism of pathogenesis of malignant tumors, cellular biological methods, with morphological, biochemical and molecular approaches utilizing cell culture, are valuable. With regard to cholangiocarcinoma, studies have been conducted using several cell lines (8-12). However, available cholangiocarcinoma cell lines are limited 
and results from these lines were predominantly intended for molecular applications. Thus, these accessible cell lines are not accompanied by detailed biochemical and morphological data sets and are insufficient for full understanding of the pathogenic mechanisms.

Previously, we established a cholangiocarcinoma cell line from a 78-year-old female patient diagnosed with extrahepatic cholangiocarcinoma. This line was designated as TK and was demonstrated to express biochemical markers, such as carbohydrate antigen (CA) 19-9, CA50 and carcino-embryonic antigen (CEA) (13). The cell line is able to be implanted into nude mice to produce xenograft animal models. However, in addition to these biochemical characterizations, morphological study is required for a greater understanding of the nature of the cell line, particularly its utilization as an experimental cholangiocarcinoma model.

A three-dimensional cell culture method was also developed with an originally-devised collagen mesh. In the mesh, cells are able to attach freely to the scaffold and proliferate without space limitation from the anchors. One application of this method is in the investigation of cell differentiation. For example, when fibroblasts were cultured three-dimensionally in the presence of demineralized bone powder, the cells produced an extracellular matrix similar to that deposited around chondrocytes $(14,15)$. This effect was confirmed by kinetic analysis of gene expression throughout the chondroinduction period (16). Furthermore, this method of cell culture is able to be combined with several bioreactors. The effects of medium perfusion $(17)$, hydrostatic pressure $(18,19)$ and exposure to low oxygen tension (20) were analyzed by this method. In addition, the culture was particularly useful for a morphological study, simulating the in vivo behavior of a malignant tumor. The method enabled individual distinction of the four representative malignant glioma cell lines used for cell biological experiments, along with their different characteristics. Behaviors associated with cell differences, migration, attachment and patterns of proliferationbecame evident only following three-dimensional cell culture (21).

The use of three-dimensional cell culture is becoming a more widespread and is being applied to a broader range of cell lines (22). However, there are few studies regarding three-dimensional cell culture of human cholangiocarcinoma cell lines. To add to the biochemical data obtained in our previous study, in the present study the morphology of the TK cell line was analyzed in three-dimensional cell culture to determine whether or not the cell line may be used for a wide range of experimental studies of cholangiocarcinoma in vitro.

\section{Materials and methods}

TK cell line and three-dimensional cell culture. TK cells were cultured with RPMI-1640 complete medium (Gibco Life Technologies Japan, Tokyo, Japan), supplemented with 15\% fetal bovine serum (Lot no. SFB30-1478, Equitech-Bio, Kershville, TX, USA), $2 \mathrm{mM}$ glutamine and $1 \mathrm{mM}$ sodium pyruvate (Gibco Life Technologies Japan). The three-dimensional culture method for the experiments was a modification of the method described by Mizuno et al (15). The scaffold material used for three-dimensional culture was a bio-absorbable, degradable gelatin. In brief, dispersed TK cells ( $1 \times 10^{4}$ cells $/ 100 \mu 1$ complete RPMI medium) were injected into the scaffolds of three-dimensional meshes and left to stand for 4-6 hours at $37^{\circ} \mathrm{C}$ in a $5 \% \mathrm{CO}_{2}$ incubator. Following the attachment of the cells to the scaffolds, the meshes were transferred onto a $10-\mathrm{cm}$ dish, immersed in $10 \mathrm{ml}$ culture medium and further cultivated for 3-20 days (21).

\section{Morphological examinations}

Phase contrast microscopy. Cell attachment and proliferation in three-dimensional culture were observed with a phase contrast microscope (CK2, Olympus Corporation, Tokyo, Japan). The meshes were directly subjected to microscopy without fixing or staining during the culture.

Light optical microscopy. Cells in the culture mesh were fixed with $10 \%$ phosphate-buffered formalin and subjected to an automatic paraffin embedding system (ETP-150CV, Sakura Finetek Japan Co., Ltd., Tokyo, Japan). Paraffin-embedded specimens were sliced into $6-\mu$-thick sections using a microtome and stained with haematoxylin and eosin. These sections were examined under a light microscope (IX71, Olympus Corporation). Images were captured using a charge-coupled device image sensor (VB-7010, Keyence Japan, Osaka, Japan).

Scanning electron microscopy (SEM). The cells attached to the mesh were fixed by treatment with $1.2 \%$ glutaraldehyde in

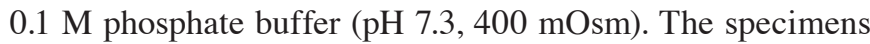
were dehydrated with a graded series of ethanol ranging from $50 \%$ through 70, 80, 90 and $100 \%$. Following further treatment with $100 \%$ iso-amylacetate, the samples were dried by a critical point dryer (Hitachi High-Technologies Corporation, Tokyo, Japan) and sprayed with Au-Pd. Cells in the mesh were examined at $15 \mathrm{kV}$ under a JSM-5800LV scanning electron microscope (JEOL Ltd., Tokyo, Japan).

Transmission electron microscopy (TEM). For transmission electron microscopy, the tissues were fixed with $2 \%$ glutaraldehyde in $0.1 \mathrm{M}$ phosphate buffer and dehydrated by serial dilution of ethanol. Subsequent to treatment with a substituting agent, the tissues were infiltrated prior to polymerization in epoxy resin and sectioning with an ultramicrotome (Leica, Vienna, Austria). Ultra-thin sections were further treated with uranyl acetate and lead citrate, and observed by the Hitachi H-7500 transmission electron microscope (Hitachi High-Technologies Corporation, Tokyo, Japan).

\section{Results}

Morphology of cultured cells in the scaffold. Following injection of dispersed TK cells into the three-dimensional mesh, the cells attached to the scaffold. Inoculated cells initially adhered to the material and then started to grow at the attached spots. Plating efficiency was not determined, as unattached cells did not remain attached to the mesh and instead diffused into the culture medium and were removed from further cultivation. After five days, proliferated cells were detected in clusters and the TK cells had aggregated and formed globoid structures (Fig. 1A). Fig. 1A obtained by phase contrast microscopy demonstrates the appearance of the cholangiocarcinoma 

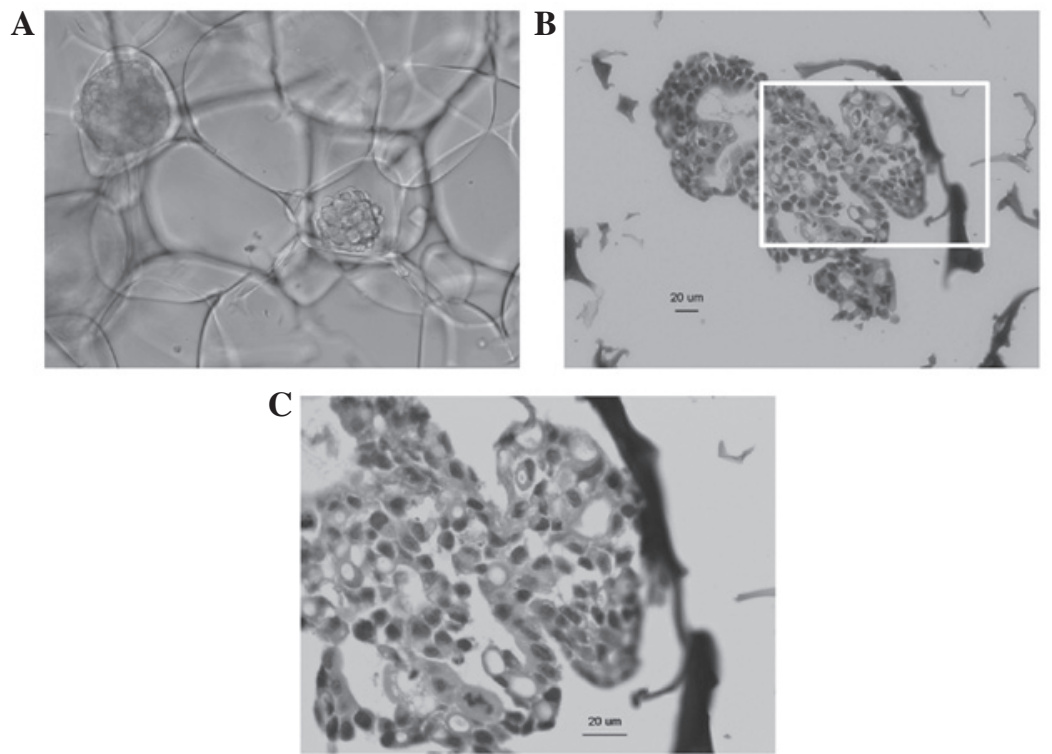

Figure 1. Three-dimensional culture of TK cells in the scaffold. (A) Appearance of the cholangiocarcinoma cells by phase contrast microscopy on day 5 of culture (magnification, x20). (B) Optical micrograph of the cholangiocarcinoma on day 14 of culture. TK cells aggregated and formed a duct-like structure at $\mathrm{x} 40$ maginifcation and at (C) $\mathrm{x} 100$ magnification.
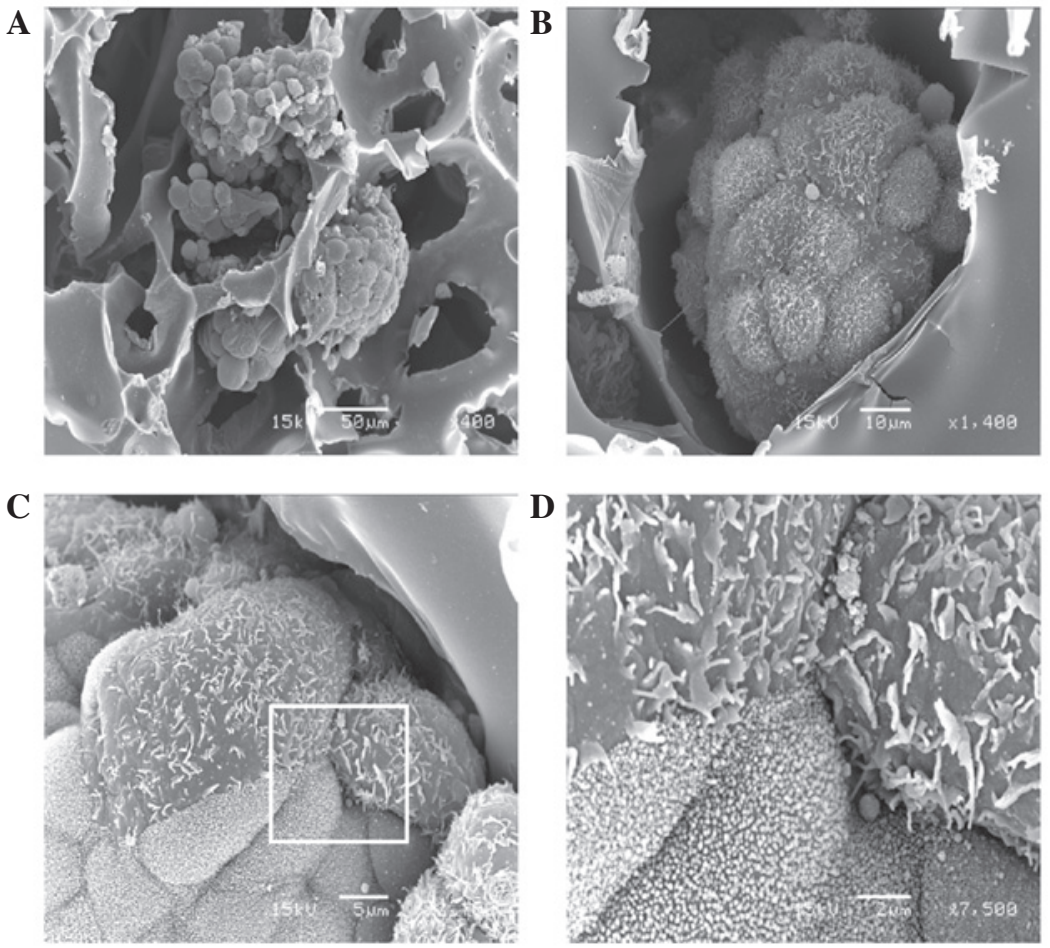

Figure 2. Scanning electron micrographs on day 10 of the three-dimensional culture. (A) TK cells attached to the scaffold and proliferated (magnification, x400). (B) Cells aggregated and formed a globoid structure. Although the cells conglomerated and formed a balloon-like structure, the boundary of each cell was relatively clear. (magnification. x1,400). (C) The surface of the cells was covered with dense microvilli or sparse plicae (magnification, x2,700). (D) Although the cultured cells originated from the same TK cell line, the cells exhibited different morphologies at the surface (magnification, $\mathrm{x} 7,500$ ).

cluster in three-dimensional culture. The structure grew relative to the culture duration. On day 14 of culture, light optical microscopy demonstrated that the TK cells had aggregated and formed duct-like assemblies (Fig. 1B). At higher magnification, cells were observed to be filled with deposits of a secretory substance in the cytoplasm (Fig. 1C). These deposits were periodic acid schiff-positive as demonstrated in a previous study (13). Fig. 1C also demonstrated the organization of the attachment of the cell to the scaffold.

SEM shows cell processes on TK cell surfaces. The cells aggregated and formed globoid shapes in the scaffold. The overall architecture was clearly demonstrated by scanning electron microscopy on day 10 of culture (Fig. 2A). The 

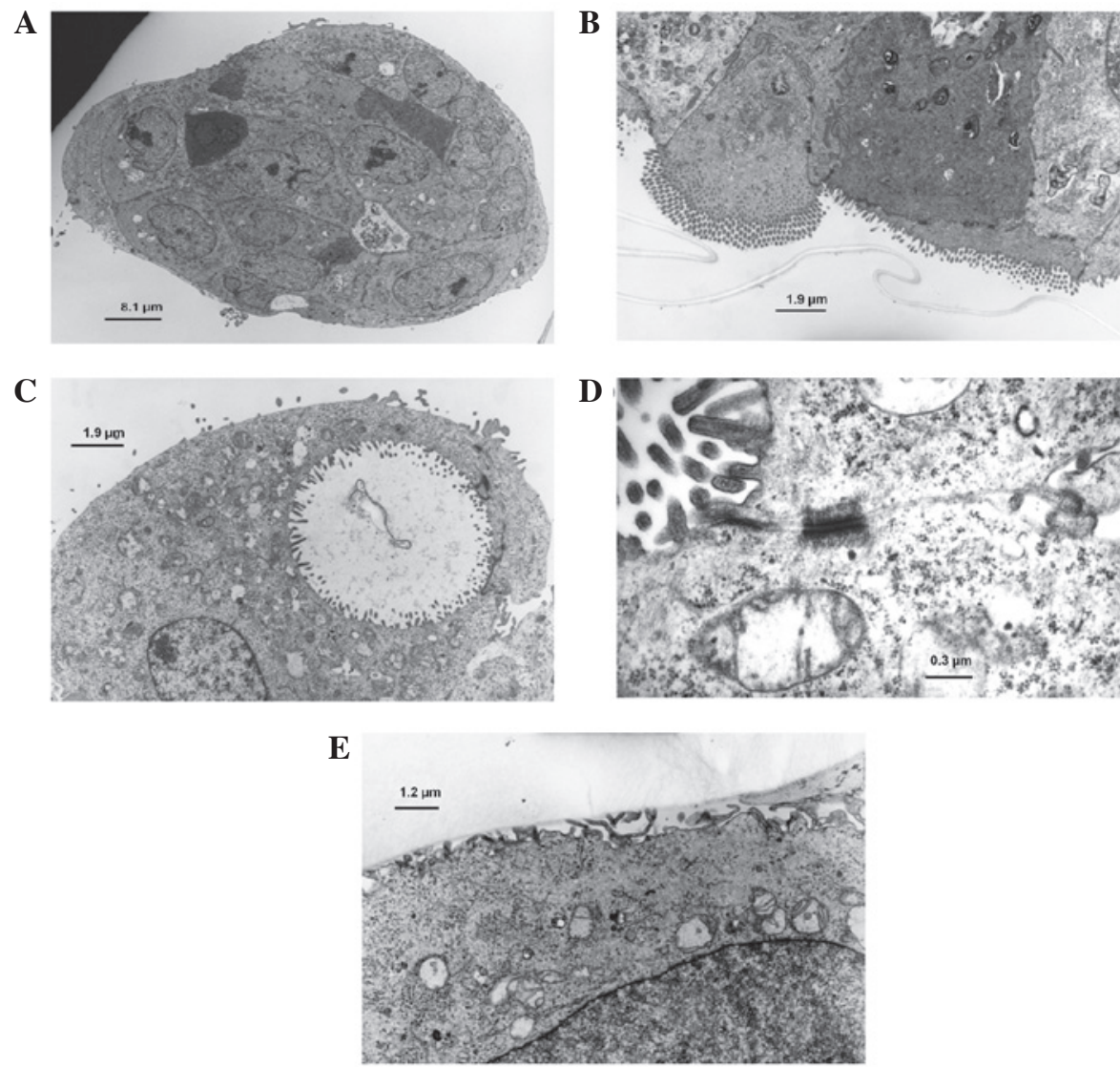

Figure 3. Transmission electron micrographs on day 7 of three-dimensional culture. (A) A cross-section of the structure. The extracellular space inside the structure is shown. (B) The surface of the structure is covered with microvilli and each cell exhibits a different electron density. (C) The cultured cell had a granular or duct-like structureand the lumen was covered with microvilli. (D) Higher magnification revealed that the structure was composed of more than one cell and a desmosome was observed between cells. (E) Attachment of the cell to the scaffold.

surface of the structure consisting of TK cells was covered with numerous floral-shaped microvilli. Closer observation revealed that the pattern was not homogeneous, despite the cells originating from the same cell line (Fig. 2B). Higher magnification of another region of the culture demonstrated that certain cells possessed relatively sparse plicae whereas others possessed dense microvilli (Fig. 2C). These two types of processes were observed on the same globoid structure; however, were segregated and distributed differently. This was confirmed by examination at higher magnification (x7,500; Fig. 2D).

TEM observation of the three-dimensional culture. A cross-section of the globoid aggregate on day 7 of the three-dimensional culture was observed by TEM (Fig. 3A), showing the internal arrangement of the cells to be semi-irregular. Cells of varying electron-density were observed in the microscopy image. Numerous microvilli were identified on the surface of the structure and also protruded into the extracellular space on the inner side of the aggregate. The cells exhibited irregularly-shaped nuclei and the endoplasmic reticula and mitochondria were not well-developed. On the outside of the structure, microvilli were observed only on the surface layer of the cells and distribution of the microvilli was demonstrated to be dense when observed under higher magnification (x5,000; Fig. 3B). When cultured three-dimensionally, certain cells formed gland-like structures and the lumen were covered by microvilli (Fig. 3C). These structures consisted of multiple cells attached to each other by a cell adherent apparatus, such as a desmosome (Fig. 3D). The scaffold demonstrated bio-adaptability and cultured cells attached to the scaffold via cell processes and/or microvilli (Fig. 3E).

\section{Discussion}

In this study, the established human TK cholangiocarcinoma cell line was cultivated three-dimensionally. Morphological observations demonstrated characteristics of cholangiocarcinoma that are not observed by ordinary two-dimensional culture. The observations of the morphological characteristics of the cultured TK cells add to the biochemical characteristics demonstrated in a previous study (13).

Cholangiocarcinoma is one of the most intractable human diseases (23). The majority of cases are inoperable and only $30 \%$ of patients qualify for surgical treatment (24). While total numbers of patients are small compared with those with more common carcinomas of the colon or lung, the rising incidence (25) and high mortality rate (26) require the development of more effective therapeutic strategies. The median survival time is $<12$ months in unresectable cholangiocarcinoma (12) and even in cases of radical resection, the recurrence rate was reported to be high (27). In the absence of PSC, curative surgical resection results in a 5-year survival rate of only $2-43 \%$ (28). Therefore, the development of an additional 
cholangiocarcinoma cell line with clearly understood biochemical and morphological characteristics may be useful for further understanding of the nature of this malignancy.

The TK cell line originated from ascites of an extrahepatic cholangiocarcinoma patient. The carcinoma of the common bile duct had diffusely invaded the liver, gallbladder and hepatoduodenal ligament. The CT scan revealed swelling of the 12th lymph node and blood analysis demonstrated elevated levels of $\gamma$-glutamyl transpeptidase, alkaline phosphatase and liver activator protein (13). The incidence of cholangiocarcinoma is relatively high in males, possibly due to a higher incidence of PSC (29); however, the patient from which the TK cell line was derived was female. Approximately $90 \%$ of patients diagnosed with cholangiocarcinoma do not have a recognized risk factor for the malignancy $(30,31)$ and in the present case, parasitic infection or exposure to toxic risk factors, such as Thorotrast, were not reported. As this cell line was established from a Japanese patient, the model may be useful for studying local specificity, such as the incidence of cases in the Japanese printing industrial sector.

Under ordinary culture, the TK cells adhered and grew on the two-dimensional surface of the culture flask or dish as previously described (13). While the majority of cells clearly attached to the culture device, certain cells were observed to accumulate or float suggesting that the cells were of ascitic origin. Monolayered TK cells were also demonstrated to frequently form a gland-like structure. However, these cells did not exhibit steric cell connections, unlike in the three-dimensional culture in the present study, which demonstrated the existence of microvilli in the luminal structures. The morphology was comparable to patterns of three-dimensional culture of various glioma cell lines (21). Numerous studies have suggested that three-dimensional cell-to-cell connections are important for proliferation, adhesion, migration, invasion and cell phenotype, and that two-dimensional cells on flat and hard plastic dishes or flasks are not representative of natural cells in living tissues or organs (22).

A number of methods have been utilized for three-dimensional cell culture. Representative examples include reconstituted basement membrane (commercially known as Matrigel) (32-33) and spheroids (35,36). Numerous other devices with a collagen scaffold are also available. One advantage of device used in the present study is its applicability as a scaffold to various types of extracellular matrix. This is due to the mesh being directly produced from a solution of numerous types of matrix. The extracellular matrix is also known to be important for morphogenesis as it is involved in the cell-to-cell connection and anchoring of cells (37). In addition, the culture may be used in combination with bioreactors and the cultured mesh may be implanted for in vivo experiments. Moreover, by labeling the cells with radioisotopes or using the release method, the culture is valuable for cytolytic or cytotoxic assays (38).

Morphological assessment of the three-dimensional culture of TK revealed steric attachments to neighboring cells with cell-adhering apparatus, such as desmosomes and scaffolds. When cultured three-dimensionally, the cells constructed globoid structures and their surface was covered with distinctive microvilli. These structures were demonstrated in gland-like structures, the lumen of which was also covered with microvilli. These observations indicate the morphology of TK cells as being cholangiocarcinomatous in origin. The results demonstrate that TK cells may be used as a model of a cholangiocarcinoma cell line in carious aspects of their morphology in addition to their previously established biochemical properties. Moreover, this culture method may be useful for elucidating the pathogenesis of cholangiocarcinoma and may be beneficial in therapeutic investigations of a number of factors, such as the sensitivity to anticancer strategies, using morphological observation. The results of the present study highlight the requirement for further analysis of the TK cell line and its potential use in the development of therapeutics.

\section{Acknowledgements}

The authors would like to thank Ms. Keiko Tomaru and Mayumi Nomura (Department of Molecular Cell Biology, Jikei University School of Medicine) for their assistance with cell culture and Ms. Hisako Arai, Emi Kikuchi and Mr. Hideki Saito (Department of Molecular Cell Biology, Jikei University School of Medicine) for the morphological images.

\section{References}

1. Faris JE and Zhu AX: Targeted therapy for biliary tract cancers J Hepatobiliary Pancreat Sci 19: 326-336, 2012.

2. Valle J, Wasan H, Palmer DH, et al: Cisplatin plus gemcitabine versus gemcitabine for biliary tract cancer. N Engl J Med 362: 1273-1281, 2010

3. Gores GJ: Cholangiocarcinoma: current concepts and insights. Hepatology 37: 961-969, 2003.

4. Khan SA, Davidson BR, Goldin RD, et al: Guidelines for the diagnosis and treatment of cholangiocarcinoma: an update. Gut 61: 1657-1669, 2012.

5. Rustagi T and Dasanu CA: Risk factors for gallbladder cancer and cholangiocarcinoma: similarities, differences and updates. J Gastrointest Cancer 43: 137-147, 2012.

6. Kubo S, Nakanuma Y, Takemura S, et al: Case series of 17 patients with cholangiocarcinoma among young adult workers of a printing company in Japan. J Hepatobiliary Pancreat Sci: Jan 13, 2014 (Epub ahead of print).

7. Kumagai S, Kurumatani N, Arimoto A and Ichihara G: Cholangiocarcinoma among offset colour proof-printing workers exposed to 1,2-dichloropropane and/or dichloromethane. Occup Environ Med 70: 508-510, 2013.

8. Meng F, Henson R, Lang M, et al: Involvement of human micro-RNA in growth and response to chemotherapy in human cholangiocarcinoma cell lines. Gastroenterology 130: 2113-2129, 2006.

9. Mott JL, Kobayashi S, Bronk SF and Gores GJ: mir-29 regulates Mcl-1 protein expression and apoptosis. Oncogene 26: 6133-6140, 2007.

10. Pignochino Y, Sarotto I, Peraldo-Neia C, et al: Targeting EGFR/HER2 pathways enhances the antiproliferative effect of gemcitabine in biliary tract and gallbladder carcinomas. BMC Cancer 10: 631, 2010.

11. Saito S, Ghosh M, Morita K, Hirano T, Miwa M and Todoroki T: The genetic differences between gallbladder and bile duct cancer cell lines. Oncol Rep 16: 949-956, 2006.

12. Selaru FM, Olaru AV, Kan T, et al: MicroRNA-21 is overexpressed in human cholangiocarcinoma and regulates programmed cell death 4 and tissue inhibitor of metalloproteinase 3. Hepatology 49: 1595-1601, 2009.

13. Watanabe M, Chigusa M, Takahashi H, Nakamura J, Tanaka H and Ohno T: High level of CA19-9, CA50, and CEA-producible human cholangiocarcinoma cell line changes in the secretion ratios in vitro or in vivo. In Vitro Cell Dev Biol Anim 36: 104-109, 2000.

14. Mizuno S and Glowacki J: Three-dimensional composite of demineralized bone powder and collagen for in vitro analysis of chondroinduction of human dermal fibroblasts. Biomaterials 17: 1819-1825, 1996. 
15. Mizuno S and Glowacki J: Chondroinduction of human dermal fibroblasts by demineralized bone in three-dimensional culture. Exp Cell Res 227: 89-97, 1996.

16. Yates KE, Mizuno S and Glowacki J: Early shifts in gene expression during chondroinduction of human dermal fibroblasts Exp Cell Res 265: 203-211, 2001.

17. Mizuno S, Allemann F and Glowacki J: Effects of medium perfusion on matrix production by bovine chondrocytes in three-dimensional collagen sponges. J Biomed Mater Res 56: 368-375, 2001.

18. Manome Y, Saeki N, Yoshinaga H, Watanabe M and Mizuno S: A culture device demonstrates that hydrostatic pressure increases mRNA of RGS5 in neuroblastoma and CHC1-L in lymphocytic cells. Cells Tissues Organs 174: 155-161, 2003.

19. Mizuno S, Tateishi T, Ushida T and Glowacki J: Hydrostatic fluid pressure enhances matrix synthesis and accumulation by bovine chondrocytes in three-dimensional culture. J Cell Physiol 193: 319-327, 2002.

20. Mizuno S and Glowacki J: Low oxygen tension enhances chondroinduction by demineralized bone matrix in human dermal fibroblasts in vitro. Cells Tissues Organs 180: 151-158, 2005.

21. Manome Y, Mizuno S, Akiyama N, et al: Three-dimensional cell culture of glioma and morphological comparison of four different human cell lines. Anticancer Res 30: 383-389, 2010.

22. Pampaloni F, Reynaud EG and Stelzer EH: The third dimension bridges the gap between cell culture and live tissue. Nat Rev Mol Cell Biol 8: 839-845, 2007.

23. Noel MS and Hezel AF: New and emerging treatment options for biliary tract cancer. Onco Targets Ther 6: 1545-1552, 2013.

24. Marsh Rde W, Alonzo M, Bajaj S, et al: Comprehensive review of the diagnosis and treatment of biliary tract cancer 2012. Part I: diagnosis-clinical staging and pathology. J Surg Oncol 106: 332-338, 2012.

25. Patel T: Cholangiocarcinoma. Nat Clin Pract Gastroenterol Hepatol 3: 33-42, 2006.

26. Farley DR, Weaver AL and Nagorney DM: 'Natural history' of unresected cholangiocarcinoma: patient outcome after noncurative intervention. Mayo Clin Proc 70: 425-429, 1995.
27. Jarnagin WR and Shoup M: Surgical management of cholangiocarcinoma. Semin Liver Dis 24: 189-199, 2004.

28. Malhi $\mathrm{H}$ and Gores GJ: Review article: the modern diagnosis and therapy of cholangiocarcinoma. Aliment Pharmacol Ther 23: 1287-1296, 2006.

29. Henson DE, Albores-Saavedra J and Corle D: Carcinoma of the extrahepatic bile ducts. Histologic types, stage of disease, grade, and survival rates. Cancer 70: 1498-1501, 1992.

30. Ben-Menachem T: Risk factors for cholangiocarcinoma. Eur J Gastroenterol Hepatol 19: 615-617, 2007.

31. Lazaridis KN and Gores GJ: Cholangiocarcinoma. Gastroenterology 128: 1655-1667, 2005.

32. Kleinman HK and Jacob K: Invasion assays. Curr Protoc Cell Biol Chapter 12: Unit 12.2, 2001.

33. Kleinman HK and Martin GR: Matrigel: basement membrane matrix with biological activity. Semin Cancer Biol 15: 378-386, 2005.

34. Marques MM, Martins MD and França CM: Effect of Matrigel on adenoid cystic carcinoma cell line differentiation. Int J Exp Pathol 87: 405-410, 2006.

35. de Ridder L, Cornelissen M and de Ridder D: Autologous spheroid culture: a screening tool for human brain tumour invasion. Crit Rev Oncol Hematol 36: 107-122, 2000.

36. Kunz-Schughart LA, Kreutz M and Knuechel R: Multicellular spheroids: a three-dimensional in vitro culture system to study tumour biology. Int J Exp Pathol 79: 1-23, 1998.

37. Kleinman HK, Philp D and Hoffman MP: Role of the extracellular matrix in morphogenesis. Curr Opin Biotechnol 14: 526-532, 2003.

38. Manome Y, Furuhata H, Hashimoto A, et al: Application of therapeutic insonation to malignant glioma cells and facilitation by echo-contrast microbubbles of levovist. Anticancer Res 29: 235-242, 2009 